\title{
Novel intra-genic large deletions of CTNNB1 gene identified in wild type desmoid-type fibromatosis
}

\author{
Chiara Colombo $^{1}$ ( ) | Milena Urbini ${ }^{2}$ ( ) | Annalisa Astolfi ${ }^{2}$ | Paola Collini ${ }^{3}$ | \\ Valentina Indio $^{2}$ | Antonino Belfiore ${ }^{3}$ | Nicholas Paielli ${ }^{3}$ | Federica Perrone ${ }^{3}$ | \\ Giuseppe Tarantino $^{2}$ | Elena Palassini ${ }^{4}$ | Marco Fiore ${ }^{1}$ | Andrea Pession ${ }^{2}$ | \\ Silvia Stacchiotti ${ }^{4}$ | Maria Abbondanza Pantaleo ${ }^{2,5}$ | Alessandro Gronchi ${ }^{1}$
}

${ }^{1}$ Department of Surgery, Fondazione IRCCS Istituto Nazionale dei Tumori, Milano, Italy

2"Giorgio Prodi" Cancer Research Center, University of Bologna, Bologna, Italy

${ }^{3}$ Department of Pathology, Fondazione IRCCS Istituto Nazionale dei Tumori, Milano, Italy

${ }^{4}$ Department of Cancer Medicine, Fondazione IRCCS Istituto Nazionale dei Tumori, Milano, Italy

${ }^{5}$ Department of Specialized, Experimental and Diagnostic Medicine, Sant'Orsola-Malpighi Hospital University of Bologna, Bologna, Italy

Correspondence

Chiara Colombo, Sarcoma Service,

Department of Surgery, Fondazione IRCCS Istituto Nazionale dei Tumori, via Venezian

1, 20133, Milano, Italy.

Email: chiara.colombo@istitutotumori.mi.it

\begin{abstract}
A wait and see approach for desmoid tumors (DT) has become part of the routine treatment strategy. However, predictive factors to select the risk of progressive disease are still lacking. A translational project was run in order to identify genomic signatures in patients enrolled within an Italian prospective observational study. Among 12 DT patients (10 CTNNB1-mutated and 2 wild type) enrolled from our institution only two patients (17\%) showed a progressive disease. Tumor biopsies were collected for whole exome sequencing. Overall, DT exhibited low somatic sequence mutation rate and no additional recurrent mutation was found. In the two wild type (WT) cases, two novel alterations were detected: a complex deletion of APC and a pathogenic mutation of LAMTOR2. Focusing on WT DT subtype, deep sequencing of CTNNB1, APC and LAMTOR2 was conducted on a retrospective series of 11 WT DT using a targeted approach. No other mutation of LAMTOR2 was detected, while APC was mutated in two cases. Low-frequency (mean reads of $16 \%$ ) CTNNB1 mutations were discovered in five samples (45\%) and two novel intra-genic deletions in CTNNB1 were detected in two cases. Both deletions and low frequency mutations of CTNNB1 were highly expressed. In conclusion, a minority of DT is WT for either CTNNB1, APC or any other gene involved in the WNT pathway. In this subgroup novel and hard to be detected molecular alterations in APC and CTNNB1 were discovered, contributing to explain a portion of the allegedly WT DT cases.
\end{abstract}

\section{KEYWORDS}

CTNNB1, deletion, desmoid-type fibromatosis, wait and see, wild type

\section{1 | INTRODUCTION}

Desmoid tumors (DT) are rare mesenchymal diseases. Despite the complete absence of metastatic capacity, DT are very infiltrative with high local recurrence rates even after complete surgical resection. In recent years, as an alternative to mutilating surgery, patient surveillance (wait and see, $W \& S$ ) has been routinely proposed as part of initial management. ${ }^{1}$ In fact, long lasting stability and spontaneous regression are not infrequent events. ${ }^{2}$ Unfortunately, there are no

Chiara Colombo and Milena Urbini contributed equally to this work. molecular or clinical predictors to guide clinicians in the daily practice, for promptly selecting patients for surgery/medical therapy or for continuous W\&S. Recently, a potential prognostic value of $\beta$-catenin has been shown in two series of surgically treated patients. A specific mutation (S45F) had a worse outcome in terms of recurrence free survival. $^{3,4}$ Moreover, the same mutation has been associated with a better response to Imatinib in patients with progressive disease. ${ }^{5}$ In order to understand the natural history of this challenging disease, two prospective European clinical trials (NCT02547831; NCT01801176) will provide new data on the role of $\mathrm{W} \& S$. 
We conducted a parallel translational study on patients enrolled in the Italian observational study in order to possibly identify genome alterations that drive aggressive behavior and to look for predictive factors using a high throughput genome approach. The assumption is that progression (in patients "observed") and recurrence (in patients "operated") represents different faces of biological aggressiveness. This could be useful in the future to personalize treatments according to the predicted aggressiveness of the disease in each patient from the beginning.

In recent years, high-throughput technologies such as nextgeneration sequencing (NGS) have been widely used to identify germline and somatic mutations or translocations in DNA that play a crucial role in cancer development and progression. In the last few years, many NGS-based studies have been carried out to provide a comprehensive molecular characterization of cancers, to identify novel genetic alterations contributing to tumorigenesis, cancer progression and metastasis, and to study tumor complexity and heterogeneity.

Specific $\beta$-catenin mutation alone may not explain entirely the specific behavior of specific subgroup that is higher risk for recurrence for S45F. Different genome alterations may be implicated in the proliferation/progression and growth arrest/regression. Despite this assumption, data available do not confirm it. In fact, Crago et al. demonstrated that mutations affecting CTNNB1 or APC occur more frequently than previously recognized (95\% vs. $80 \%-85 \%$ ), and designation of wild-type genotype (WT) is largely determined by sensitivity of detection methods. ${ }^{6}$ In fact, CTNNB1 WT tumors (determined by NGS) may have genomic alterations associated with Wnt activation (chromosome 6 loss/BMI1 mutation), supporting Wnt/bcatenin activation as the common pathway governing DT initiation.

Even though the majority of DT are karyotypically stable tumors, recurrent copy number alterations have been previously described, including loss of chromosome 5 and 6 in $5 \%-11 \%$ of cases and gain of chromosome 8 and 20 in a minority of patients. ${ }^{7-10}$ Loss of chromosome arm 5q, involving APC, was detected in tumors from both patients with and without FAP. ${ }^{7}$ The meaning of these events in the pathogenesis of the disease has never been elucidated. The aim of this study was to characterize the molecular events beyond Wnt/beta catenin pathway in a prospectively enrolled patients under W\&S and in a subgroup of DT samples assigned by Sanger sequencing to the WT subclass.

\section{2 | MATERIALS AND METHODS}

\section{1 | Case selection}

Twelve patients with primary sporadic naïve DT enrolled in the prospective study were included in this translational study. Recurrent, pre-treated and FAP patients were excluded. A specific informed consent was obtained. Patients, tumors and molecular data are listed in Table 1. DT fresh frozen (FF) samples from enrolled patients who have been biopsied at our institution were collected for translational studies. Frozen samples and blood samples were available for the analysis. In addition, 11 FFPE samples from patients previously operated for WT DT were analyzed. Patients, tumors and molecular data are listed in Table 2. The study was approved by the Independent Ethics Committee of Fondazione IRCCS Istituto Nazionale dei Tumori (Approval Number INT 182/13).

\section{2 | DNA extraction}

DNA was extracted from FF tumors and from FFPE samples using, respectively, QIAamp DNA mini Kit (Qiagen, Milan, IT) and DNA micro Kit (Qiagen), following manufacturer's instructions. DNA was quantified using Quant-it PicoGreen dsDNA assay (Thermo Fisher, Monza, IT).

\subsection{Whole exome sequencing}

Libraries were synthesized with Truseq Rapid Capture Exome Kit (Illumina) following manufacturer's recommendations. Briefly, genomic DNA (50 ng for FF and 100 ng for FFPE samples) was tagged and fragmented by the Nextera transposome technique to an average library size of $290 \mathrm{bp}$. DNA libraries were then denatured to singlestranded DNA and hybridized to biotin-labeled 80-mer probes designed to enrich exonic regions, then eluted from magnetic beads and amplified. Libraries were quality-checked and sized with Agilent DNA High sensitivity chips on the Bioanalyzer 2100 (Agilent Technologies, Taiwan), then quantified using a fluorometric assay (Quant-it PicoGreen assay). Then, libraries were amplified and ligated to the flowcell by bridge PCR, and sequenced at $2 \times 80$ bp read length using Illumina NextSeq500 platform (Illumina).

\section{4 | Bioinformatic analysis}

For WES data, after demultiplexing and FASTQ generation, the paired-end reads were trimmed using AdapterRemoval (https:// github.com/MikkelSchubert/adapterremoval) with the aim of removing stretches of low quality bases $(<\mathrm{Q} 10)$ and adapters present in the sequences. The sequences were then aligned on human reference genome HG19 (http://genome.ucsc.edu) with BurrowsWheeler Aligner (http://bio-bwa.sourceforge.net/); the PCR and optical duplicates were removed and Genome Analysis Toolkit (https://software.broadinstitute.org/gatk) was used to local realign, recalibrate and call the ins/del variants (HaplotypeCaller function), while point mutations were identified with the algorithm Mutect (https://www.broadinstitute.org/cancer/cga/mutect). Single nucleotide variants (SNV) and ins/del were annotated using Annovar (http://annovar.openbioinformatics.org); non-synonymous and nonsense SNV, frameshift/non-frameshift ins/del and splice site mutations were selected with a threshold read depth $\geq 15 \times$ and a variant allele frequency $\geq 0.2$. A lower variant allele frequency threshold was set to $\geq 0.03$ to detect low frequency mutations exclusively on CTNNB1 gene. All the variants were filtered in order to select novel or rare events (frequency in the population $<1 \%$ ) basing on database of human variability dbSNP (http://www.ncbi. nlm.nih.gov/SNP), 1000 genomes (http://www.1000genomes.org), ExAC (http://exac.broadinstitute.org), and EVS (http://evs.gs. washington.edu/EVS). In-depth evaluation of high confidence somatic variants was performed by verifying the presence of 
TABLE 1 Molecular and clinical data of DT samples from prospective study

\begin{tabular}{|c|c|c|c|c|c|c|c|}
\hline$\#$ \#ID & $\begin{array}{l}\text { CTNNB1 } \\
\text { status }\end{array}$ & $\begin{array}{l}\text { No of somatic } \\
\text { mutations }\end{array}$ & $\begin{array}{l}\text { Mutational burden } \\
\text { (somatic mutations/Mb) }\end{array}$ & Other somatic events & Anatomical site & $\begin{array}{l}\text { Age at } \\
\text { diagnosis (years) }\end{array}$ & $\begin{array}{l}\text { Shifting to } \\
\text { active treatment }\end{array}$ \\
\hline W\&S\#1 & p.T41A & 6 & 0.168 & NRP2 p.R572Q & Abdominal wall & 32 & $\ldots$ \\
\hline W\&S\#2 & p.T41A & 6 & 0.168 & LPA p.Y1728H & Extremity & 28 & $\ldots$ \\
\hline W\&S\#3 & p.T41A & 5 & 0.143 & $\ldots$ & Abdominal wall & 31 & $\ldots$ \\
\hline W\&S\#4 & p.T41A & 7 & 0.204 & $\ldots$ & Abdominal wall & 47 & $\ldots$ \\
\hline W\&S\#5 & p.S45F & 9 & 0.255 & $\ldots$ & Abdominal wall & 26 & Yes \\
\hline W\&S\#7 & p.T41A & 12 & 0.337 & RELN p.S2062K & Abdominal wall & 42 & $\ldots$ \\
\hline W\&S\#8 & p.S45F & 12 & 0.348 & $\ldots$ & Extremity & 45 & Yes \\
\hline W\&S\#9 & WT & 13 & 0.363 & LAMTOR2 p.V92M & Abdominal wall & 39 & $\ldots$ \\
\hline W\&S\#10 & p.T41A & 14 & 0.392 & EGR2 p.A358s & Abdominal wall & 36 & $\ldots$ \\
\hline
\end{tabular}

alternate allele on the normal counterpart and manually visualizing each variation with the tview function of Samtools. The oncological relevance and the effect at protein level of each mutation was evaluated considering COSMIC database (http://cancer.sanger.ac.uk/ cosmic) and SNPeff predictor. Moreover, based on WES data, the analysis of copy number variations (either amplifications or large deletions) were performed making a consensus between Control FREEC (http://boevalab.com/FREEC) and ADTEX (http://adtex. sourceforge.net) with paired tumor/matched normal samples. A filtering procedure was applied taking into account the uncertainty value given by Control FREEC (<80\%) and the polymorphic copy number variants from the Database of human Genomic Variants (http://dgv.tcag.ca/dgv/app/home). Finally, for complex intragenic rearrangements detection, we selected all the unmapped reads from bam file and we performed local alignment with nucleotide BLAST (https://blast.ncbi.nlm.nih.gov) using the exon 3 sequence of CTNNB1 as target. Local alignment with 0 mismatches and involving at least 50 nucleotides were kept supposing that they could support a large deletion or insertion. This set of sequences were manually investigated and realigned against the full gene sequence in order to confirm the presence of breakpoint within the CTNNB1 gene.

\section{5 | Amplicon sequencing}

A custom amplicon sequencing panel targeting the whole coding sequence of CTNNB1, APC, and LAMTOR2 was designed with Design Studio software (Illumina). Considering the high degradation of FFPE DNA, we used a dual strand approach (able to discriminate reads produced from positive or negative strand of DNA and then to identify FFPE artifact) with a mean amplicon length of $175 \mathrm{bp}$.

30 and $10 \mathrm{ng}$ of DNA extracted from, respectively, 11 FFPE and 1 fresh CTNNB1-WT DT were used for library synthesis with Truseq Custom Amplicon Low Input Dual Strand (Illumina), following manufacturer's instructions. Briefly, for each region of interest, two custom probes were hybridized and elongated copying target
DNA. The two elongation products were then ligated and amplificated adding illumina adapters and sequencing primers. Libraries were then quantified using Quant-it PicoGreen dsDNA, normalized and pooled. $10 \mathrm{pM}$ of pooled libraries were sequenced on a Micro V2 flowcell on Miseq platform (Illumina) reaching an average depth of 1938x. Demultiplexing and alignment was performed with Miseq Reporter 2.6 (Illumina) using banded Smith-Waterman algorithm. Variants were identified using somatic variant caller and annotated using Variant studio 3.0 (Illumina). Variants detected in only one strand were considered as FFPE artifacts and were excluded from the analysis.

\subsection{RNA extraction and reverse-transcription}

RNA was extracted from FFPE samples using RecoverAll Total Nucleic Acid Isolation Kit (Thermo Fisher). RNA was quantified through UV absorbance. 500 ng of RNA was reverse-transcribed using SuperScript IV Reverse Transcriptase (Thermo Fisher) following manufacturer's instructions.

\section{7 | PCR and Sanger sequencing}

For APC large deletion detection, several primers covering the entire exon 15 were designed. LAMTOR2 sequencing was performed through Sanger sequencing, using primers covering the entire coding region of the gene. All PCR products were then purified and loaded onto an ABI 3730 Genetic Analyzer (Applied Biosystems, Monza, Italy) for Sanger Sequencing. To screen for CTNNB1 intragenic deletions, several primers amplifying short fragments spanning the genomic region from exon 2 to exon 4 of CTNNB1 were designed. While for FF a single amplicon covering the entire region from exon 2 to exon 4 was generated and sequenced, to overcome the high degradation of FFPE samples several short amplicons were produced and screened for aberrant amplicon length or sequenced. All primers used are listed in Supporting Information, file 1. 


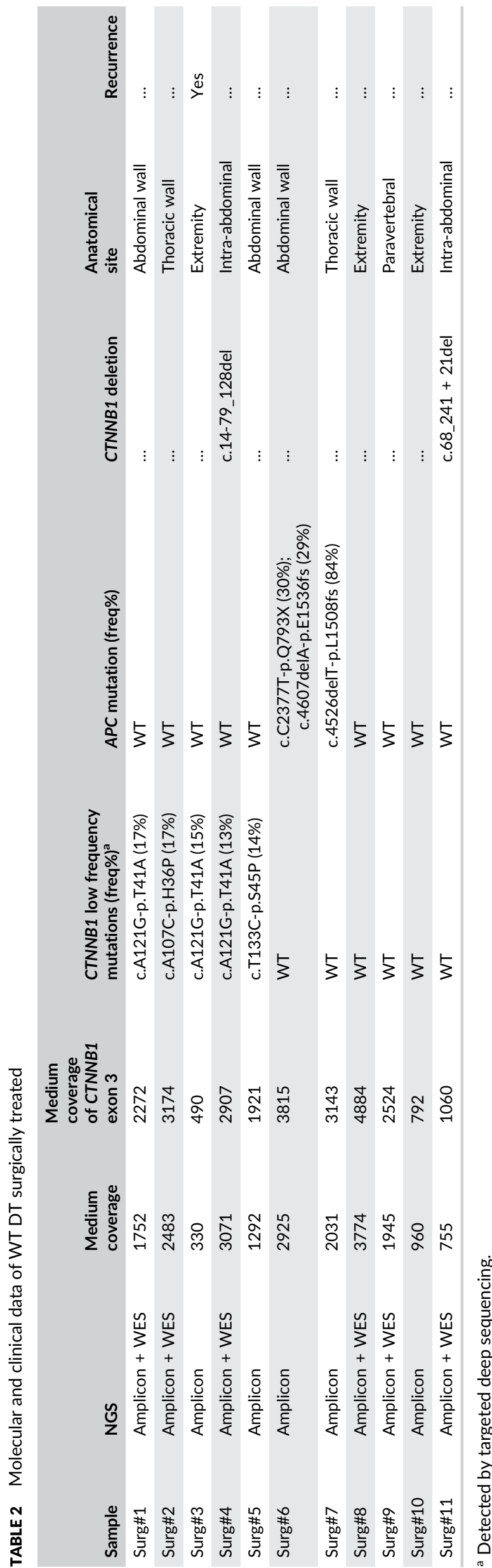

For cDNA amplification CTNNB1 primers 252F and 406R were used. Prior to Sanger sequencing, amplicons shorter than $150 \mathrm{bp}$ were cloned in a pcDNA3.1 cloning vector (TOPO-TA cloning, Thermo Fisher).

\section{3 | RESULTS}

\section{1 | Mutational landscape of DT}

FF tumors and normal counterpart of 12 DT were collected for WES analysis: 10 cases carried CTNNB1 mutations (8 T41A and 2 S45F) while 2 were classified as CTNNB1 WT DT.

All cases showed a generally stable genome, with no relevant CNV detected with the exception of one copy loss of q arm of chromosome 6 in W\&S\#6 (data not shown). Globally, all cases exhibited low somatic mutation rate, with a median value of 0.31 mutations per megabase (Table 1). Full list of somatic mutations detected is available in Supporting Information, file 2.

CTNNB1 mutant samples carried no recurrent mutational event. However, few relevant private mutations could be detected, as a STOP gain mutation of SETDB1 (p.R495X) inW\&S\#11, and missense mutations in NRP2, RELN, LPA, EGR2 and PDX1, respectively, in W\&S\#1, \#7, \#2, \#10, and \#11 (Table 1).

Of the two WT DT, W\&S\#6 harbored a complex alteration of $A P C$ involving a large deletion and acquisition of several somatic SNV. In particular, sequencing of this case unveiled the presence of five somatic mutations in the same region of exon 15 of APC (Table 1). To exclude sequencing artifact or misalignment, we sequenced a long PCR product covering almost the entire length of exon 15 (6500 bp), confirming the presence of the four somatic mutations associated to a large inframe deletion of $924 \mathrm{bp}$ in the upstream part of the exon (Figure 1).

On the contrary, W\&S\#9 did not show any alterations of genes in the WNT pathway. However, of the mutations identified, a missense mutation (p.V92M) of LAMTOR2, a gene involved in mTORC1 activation, was detected and predicted as pathogenic by three bioinformatics tools (Table 1 and Supporting Information, file 2).

\subsection{Analysis of WT DT}

To further characterize the molecular background of WT DT and to look for LAMTOR2 recurrent mutation in WT DT, FFPE tissues of 11 CTNNB1 WT DT surgically treated were collected and analyzed through NGS.

First of all, deep sequencing of CTNNB1, APC, and LAMTOR2 was performed in 12 WT cases (W\&S\#9 and 11 FFPE samples) reaching a mean coverage of 1938x. LAMTOR2 mutations were not detected in other cases, but APC mutations were identified in two cases among the surgically treated CTNNB1 WT cases: Surg\#6 carried two truncating mutations ( $\mathrm{p}$. Q793X and p.E1536fs) while Surg\#7 harbored a homozygous p.L1508fs. Moreover, through deep sequencing of CTNNB1, we unveiled the presence of five cases (Surg\#1-5) carrying low frequency mutations of CTNNB1 ranging from $13 \%$ to $17 \%$ of the altered allele (Table 2 and Supporting Information, file 3). 
A

$\mathrm{APC} \rightarrow$

Exon 15

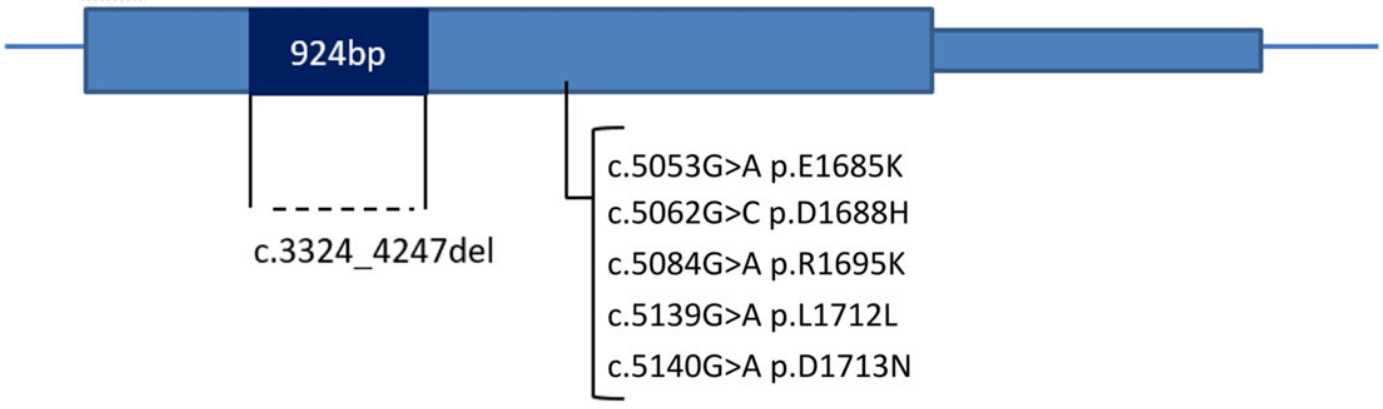

B

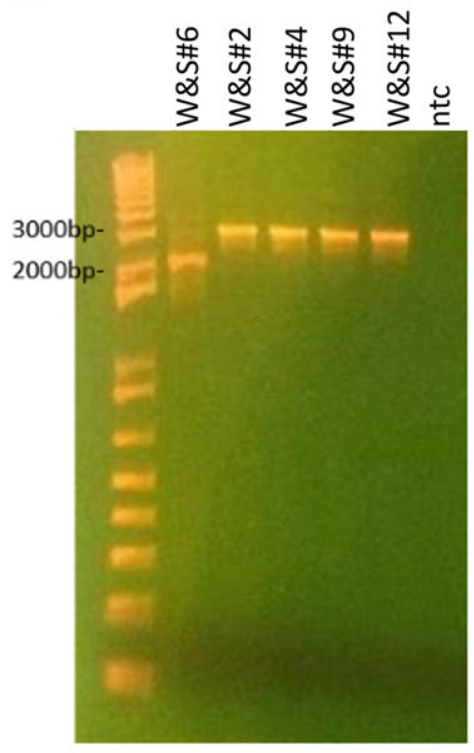

C
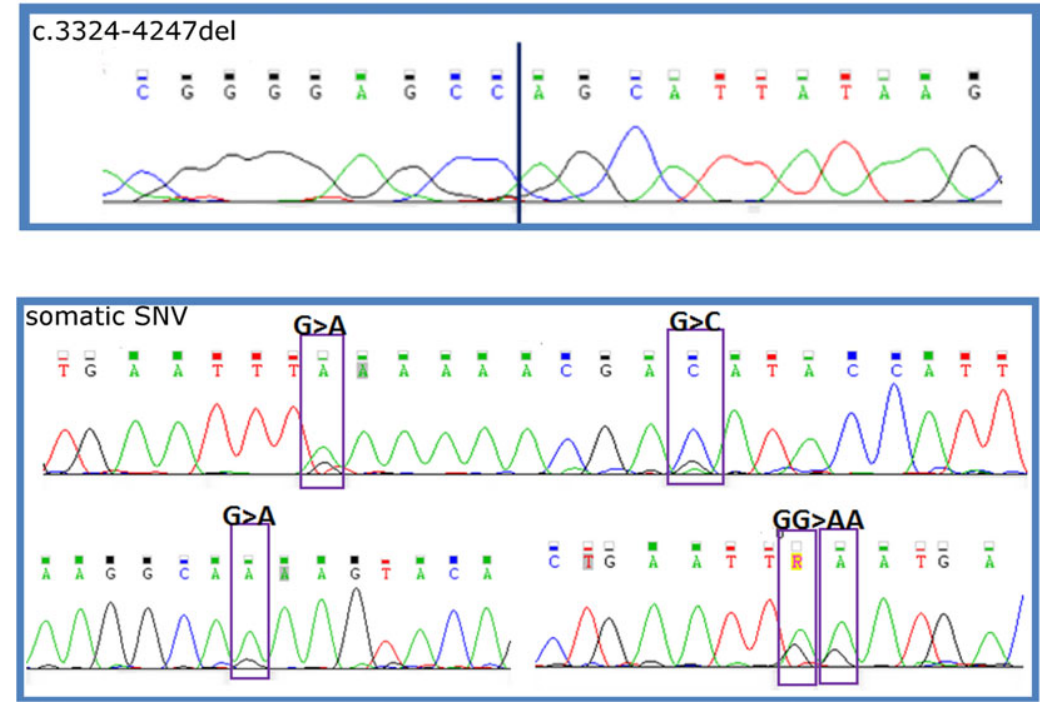

FIGURE 1 Complex APC alteration identified in W\&S\#6. (A) Schematic representation of exon 15 APC with all mutations identified in W\&S\#6. In dark blue is indicated the 924 bp deletion and a bar indicated the position of the five somatic SNV detected. (B) Identification of the large exon 15 deletion of APC through long PCR. (C) Validation through Sanger method of the breakpoint sequence, caused by the deletion of APC, and of the 5 SNV downstream. All alterations were carried by the same allele [Color figure can be viewed at wileyonlinelibrary.com]

Then, we attempted to perform WES on the surgically treated DT. However, due to low quality of FFPE DNA, we were able to synthetize WES libraries in only 6 out of 11 cases (three WT and three with low frequencies CTNNB1 mutations). Adjusting the filtering parameters of WES analysis, we validated the presence of low frequency mutation of CTNNB1 in the predicted three cases (data not shown).

\section{3 | Identification of CTNNB1 intra-genic deletions}

Since it is known that DT tumors could carry a variety of complex alterations in CTNNB1, including clonal mutations, we decided to further investigate CTNNB1 in WT cases, with the aim to detect all possible complex or cryptic mutational events.

Starting from WES data of the 6 FFPE cases (Table 2), we searched among unmapped reads for sequences encompassing exon 3 of CTNNB1. Interestingly, in two samples we detected reads supporting intragenic breakpoint events inside exon 3 of CTNNB1 (See Supporting Information, file 4). In particular, in Surg\#11 a breakpoint between exon 3 and intron 3 supported by 9 reads was detected while in Surg\#4 it was possible to identify 12 reads supporting a breakpoint between intron 2 and exon 3 (Figure 2A and Supporting Information, file 4). These findings led us to hypothesize the presence of intragenic deletions of CTNNB1 in WT DT, difficult to detect by canonical WES analysis pipelines.

To validate and to exclude the presence of other deleted cases, we performed a screening of all DT of this study through serial amplification of CTNNB1 using several primers pairs covering the genomic region between exon 2 and exon 4 (primer positions are shown in Figure 2A). We validated the actual presence of two different intragenic large deletions occurring in the two samples, Surg\#4 and Surg\#11, respectively of 192 and 195 bp (Figure 2B). No other samples tested (W\&S or FFPE cases) were shown to carry intragenic large deletions (data not shown). By PCR and Sanger sequencing, we confirmed the position of the two breakpoints. In Surg\#11 the deletion c.68_241 + 21del disrupted exon 3 after $54 \mathrm{bp}$, joining it with intron 3 sequence $21 \mathrm{bp}$ after the donor splice site. In Surg\#4 the deletion c.14-79_128del occurred between intron 2 and the middle of exon 3, right after AA T41 of the protein 
A

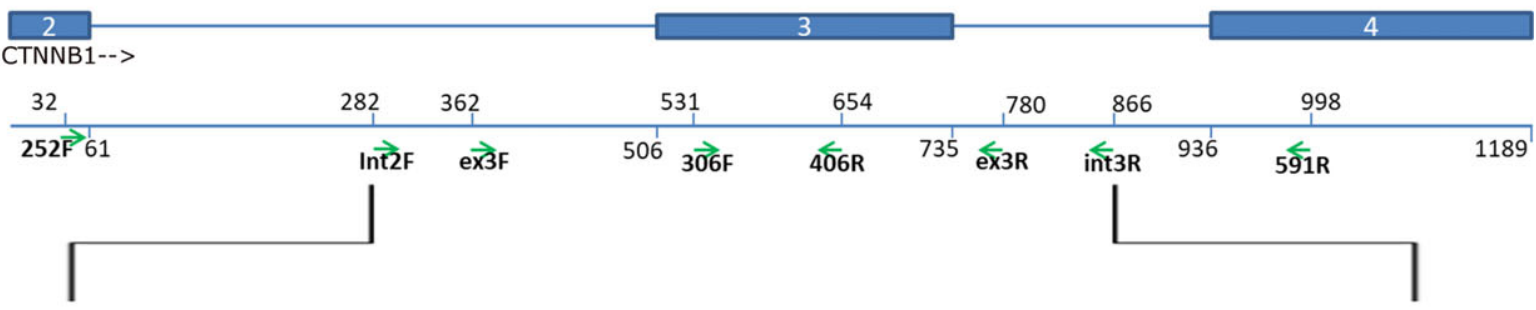
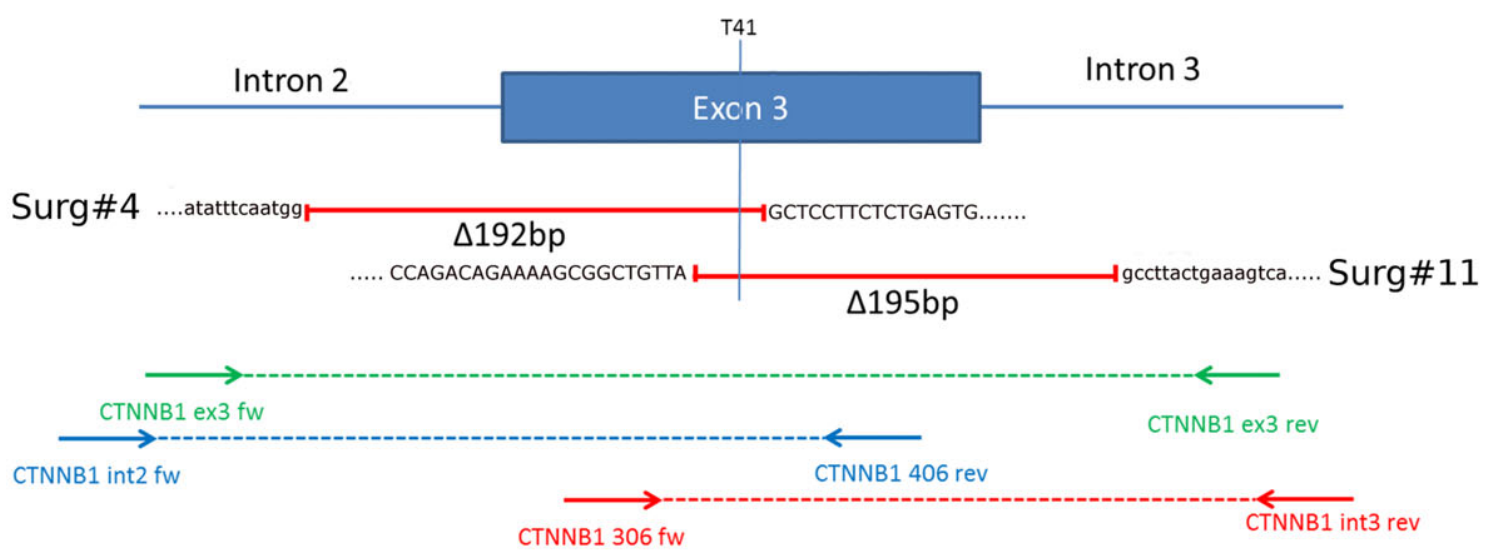

B
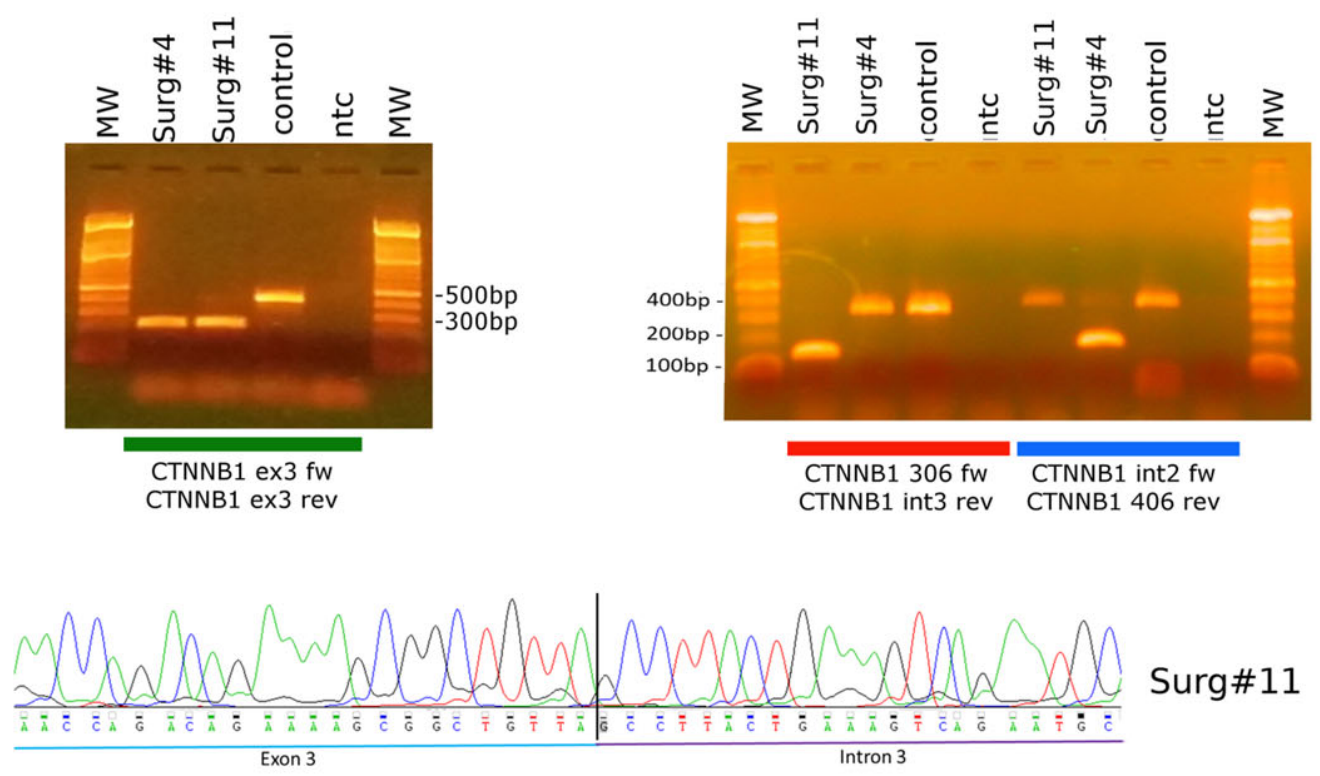

Surg\#11

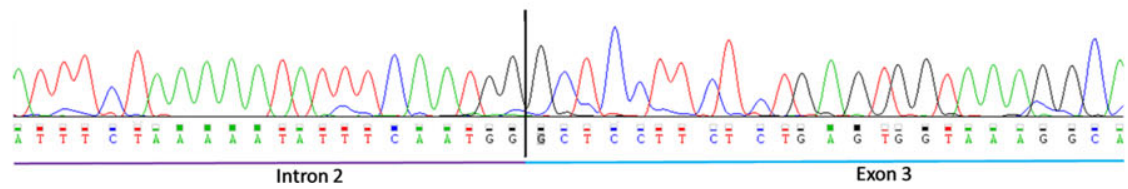

\section{Surg\#4}

FIGURE 2 Identification of large intragenic deletions of CTNNB1 in two DF tumors. (A) Schematic representation of the two deletions, identified through in-depth analysis of WES data. All primers used for validation and screening are indicated as arrows. Light blue boxes are CTNNB1 exons, light blue lines are intronic sequences, red lines represented the two deletions occurred. Positions of the mutational hotspot T41 is indicated. (B) Validation of CTNNB1 deletions through PCR. In the left panel, PCR performed with primer pairs covering the entire exon 3 detected both deletion types. Only the deleted band was detected in Surg\#4 and \#11, probably due to preferred amplification of the shorter amplicon. In the right panel amplification was performed with two primers pairs, each specific for the deletion of Surg\#4 or \#11. Primer pair 306_fw and int3_rev could detect case \#11 deletion, while in case \#4 it amplified only the WT allele. Primer pair int4_fw and 406_rev could detect case \#4 deletion, while in case \#11 it amplified only the WT allele. These findings demonstrated that both deletion event was heterozygous. (C) Chromatograms showing breakpoints of the two deletions of Surg\#4 and \#11 [Color figure can be viewed at wileyonlinelibrary.com] 
A

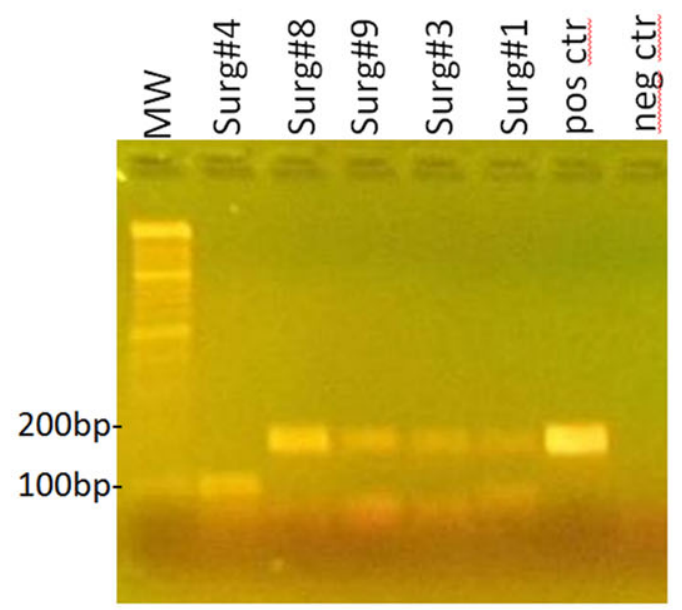

B

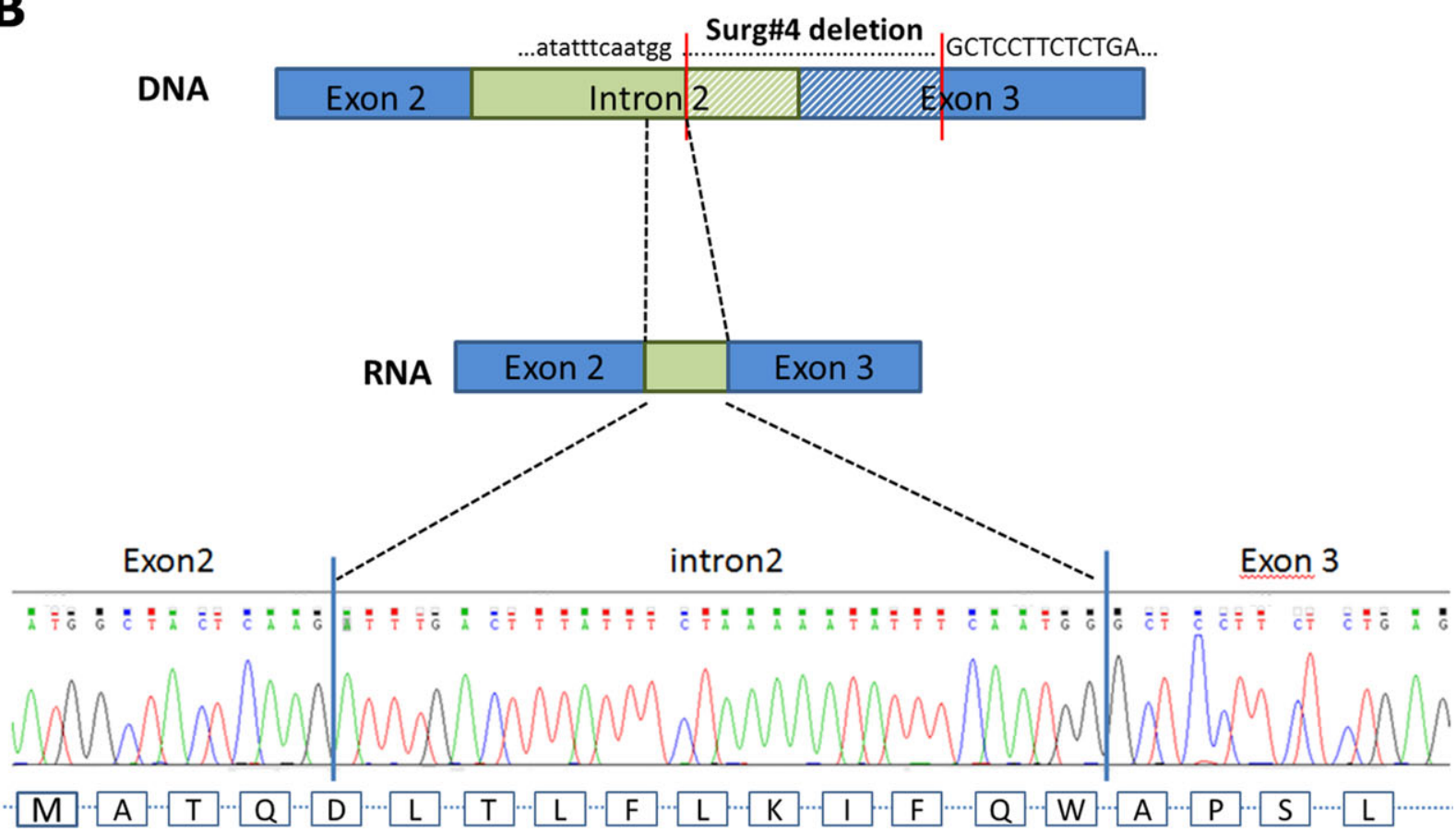

FIGURE 3 mRNA expression of CTNNB1 mutations. (A) RT-PCR of RNA extracted from 5 surgically treated DT. Primers used are CTNNB1 252F on exon 2 and 406R exon 3. Amplicons of about 200 bp corresponded to CTNNB1 full-length allele. In Surg\#4 an amplicon of about 100 bp was detected, indicating the presence of the deleted allele. (B) $100 \mathrm{bp}$ amplicon of Surg\#4 was cloned into a pcDNA3.1 plasmid and sequenced in Sanger method using primers inside the vector. In the upper side of the panel a schematic representation of the deleted isoform of CTNNB1 is shown. Blue boxes represent coding exons 2 and 3, in green intron 2, and dashed lines indicate the deleted region of Surg\#4. In the lower part, chromatogram demonstrating the inclusion of part of intron 2 in mRNA sequence is shown. This led to the retention of the coding frame of the deleted CTNNB1 isoform. (C) Chromatogram showing expression of a low frequency p.T41A mutation at mRNA level. In Surg\#3, even if only 15\% of tumor DNA carried the alteration, G allele was higher expressed than the WT and could be detected by Sanger method [Color figure can be viewed at wileyonlinelibrary.com]

(Figure 2C). Peculiarly both deletions led to the loss of the T41 codon hot spot of mutation, also in Surg\#4 where a clonal T41A was detected in the other allele.

\subsection{Expression of mutant CTNNB1}

We then evaluated whether all these different types of alterations of CTNNB1 were actually expressed. RNA extracted from 5 cases of FFPE
DT was reverse-transcribed, amplified, and sequenced with Sanger method. Peculiarly, in Surg\#4 we were able to detect the expression of the deleted allele of CTNNB1 (Figure 3A) and we unveiled the effective sequence of this truncated isoform at cDNA level. Interestingly in this case, a new acceptor splicing site was created right upstream of the intronic breakpoint, leading to a chimeric exon 3 composed by 32 bp of intron 2 joined with the remaining 114 bp of exon 3 . This event allowed the retention of the coding frame, enabling the translation of a functional 
CTNNB1 protein (Figure 3B). Finally, through cDNA sequencing of a low frequency CTNNB1 mutated sample (Surg\#3), we demonstrated that the T41A allele, even if it was carried by only $15 \%$ of cells, was the prevalent allelic isoform expressed with respect to the WT allele (Figure 3C).

\section{4 | DISCUSSION}

Sporadic DT are characterized by a low load of mutational events, except for the major molecular alterations (exon 3 CTNNB1 mutation) in the vast majority of cases. In this small series, the specific type of mutation did not seem to influence the clinical course: however, the two patients with CTNNB1 S45F mutation were shifted to an active treatment. This is a small-sized cohort and the results of the prospective European studies on a W\&S approach will convey the results through an adequately powered series.

With the aim to better characterize DT, we analyzed a prospective cohort of patients going through $W \& S$ observational protocol. Through whole exome sequencing, we confirmed the presence of specific CTNNB1 mutations in 10 patients, as already seen with Sanger sequencing. In the remaining two CTNNB1 WT samples, a complex $A P C$ mutation was discovered in one case and in the other no somatic alteration affecting WNT/CTNNB1 pathway was found. Two APC mutated cases were also detected among the 11 retrospective WT DT collected. Peculiarly all these mutations, together with the one seen in the W\&S case, occurred in exon 15 of APC, according to the previous findings that indicated the $5^{\prime}$ part of the gene as the most frequent target of mutation, especially in association with FAP disease. ${ }^{11} A P C$ is known to be affected by several types of mutation, from SNV to gross and complex insertions or deletions. Moreover, large submicroscopic deletions were reported in FAP patients. ${ }^{11,12}$ In this series, we detected a novel complex alteration of APC, composed of an intragenic deletion of part of exon 15 and the acquisition of 5 somatic mutations, adding a further layer of complexity in the characterization of $A P C$ mutational status.

In the W\&S cohort, only one case could be considered a truly WT DT. In a recent paper, Crago et al. described for the first time the presence of CTNNB1 mutated cases with low mutant allele frequency not detectable by traditional sequencing and considered as WT DT. ${ }^{6}$ In our retrospective series of WT DT, through deep sequencing technique we were able to detect CTNNB1 low frequency mutations in 5 out of 11 CTNNB1 WT cases (45\%). Despite being low frequency mutations, we demonstrated that these events were prevalently expressed, and easier to be detected at the mRNA level, since the mutated allele of CTNNB1 was much more expressed than the WT allele. However, the pathogenetic relevance of this low load of CTNNB1 mutation needs further validation. We may speculate that it can represent a favorable evolution towards a spontaneous regression. In fact, the favorable course of WT DT was already shown in different retrospective series of patients treated with surgery supporting the fact that WT may have a more indolent course. ${ }^{4,13,14}$ The two European prospective study will provide new insight on this hypothesis.

Interestingly, searching for complex mutational events occurring in CTNNB1, we discovered for the first time intra-genic deletions in DT. In particular, we unveiled the presence of two different large deletions, of about $190 \mathrm{bp}$, involving exon 3 of CTNNB1 in two DT cases. Moreover, we demonstrated that the deleted CTNNB1 was expressed and that it retained the coding frame for protein translation. Only few reports describing similar deletion of CTNNB1 in hepatoblastoma and in small bowel carcinomas are already present in literature. ${ }^{15-17}$ Interestingly, these types of deletions could be of different lengths, from 24 to $660 \mathrm{bp}$, and they could encompass the region between exon 2 to exon 4 of CTNNB1. ${ }^{16}$ Nevertheless, also in these other tumors, these intra-genic deletions were described to be in frame, leading to the production of an activated form of CTNNB1 that can localize to the nucleus. ${ }^{17}$

Unfortunately, these types of deletions are hard to be detected by high throughput techniques, since they are too large for conventional NGS analysis pipelines and too small for CGH workflow. Moreover, working on fragmented DNA, as those extracted from FFPE tissue, it is not easy to perform a PCR screening of the region. Due to these limitations, we could not exclude that other deleted cases could be present in the retrospective cohort analyzed. Therefore, even if we have detected only 2 deleted cases, the frequency of these type of alterations could be higher, thus possibly contributing to explain at least a portion of the allegedly WT DT cases. Additionally, it must be noted that one of the CTNNB1 deleted cases carried also a low allele frequency T41A mutation suggesting a high level of heterogeneity in the tumor never described so far in DT.

In conclusion, novel molecular alterations in APC and CTNNB1 were identified. A minority of DT remained WT for either CTNNB1, $A P C$ or any other gene involved in the WNT pathway. Particular attention should be paid to the intra-genic deletions of CTNNB1 that, for the complexity of detection, could have been underestimated in DT until now. These molecular findings and their clinical meanings deserve further investigation in larger series.

\section{ACKNOWLEDGMENTS}

This work has been financially supported by DTRF grant (CC). The financial support from Ministero della salute, Ricerca Finalizzata 2009 (RF-2009-1511297, AG) and by Fondazione Isabella Seràgnoli, Bologna, Italy (MAP) are gratefully acknowledged.

\section{CONFLICT OF INTEREST}

We declare that we have no conflict of interest.

\section{ORCID}

Chiara Colombo (D) http://orcid.org/0000-0001-8031-5528

Milena Urbini (iD http://orcid.org/0000-0002-3364-9098

\section{REFERENCES}

1. Kasper B, Baumgarten C, Garcia J, Bonvalot S, Haas R, Haller F, Hohenberger P, Penel N, Messiou C, van der Graaf WT, Gronchi A, Bauer S, Blay JY, van Coevorden F, Dileo P, Dürr HR, Fiore $M$, Grünwald V, Jones R, Judson I, Kettelhack C, Kopeckova K, Lazar A, Lindner LH, Martin-Broto J, Rutkowski P, Stacchiotti S, Stoeckle E, Valverde C, Verhoef K, Wardelmann E, Wartenberg M. An update on the management of sporadic desmoid-type fibromatosis: a European Consensus Initiative between Sarcoma PAtients EuroNet (SPAEN) and European Organization for Research and Treatment of Cancer 
(EORTC)/Soft Tissue and Bone Sarcoma Group (STBSG). Ann Oncol. 2017;28:2399-2408.

2. Fiore M, Rimareix F, Mariani L, Domont J, Collini P, Le Péchoux C, Casali PG, Le Cesne A, Gronchi A, Bonvalot S. Desmoid-type fibromatosis: a front-line conservative approach to select patients for surgical treatment. Ann Surg Oncol. 2009;16:2587-2593.

3. Lazar AJF, Tuvin D, Hajibashi S, Habeeb S, Bolshakov S, Mayordomo-Aranda E, Warneke CL, Lopez-Terrada D, Pollock RE, Lev D. Specific mutations in the beta-catenin gene (CTNNB1) correlate with local recurrence in sporadic desmoid tumors. Am J Pathol. 2008;173:1518-1527.

4. Colombo C, Miceli R, Lazar AJ, Perrone F, Pollock RE, Le Cesne A, Hartgrink HH, Cleton-Jansen A-M, Domont J, Bovée JVMG, Bonvalot S, Lev D, Gronchi A. CTNNB1 45F mutation is a molecular prognosticator of increased postoperative primary desmoid tumor recurrence: an independent, multicenter validation study. Cancer. 2013;119:3696-3702.

5. Kasper B, Gruenwald V, Reichardt P, Bauer S, Hohenberger P, Haller F. Correlation of CTNNB1 mutation status with progression arrest rate in RECIST progressive desmoid-type fibromatosis treated with imatinib: translational research results from a phase 2 study of the German Interdisciplinary Sarcoma Group (GISG-01). Ann Surg Oncol. 2016;23:1924-1927.

6. Crago AM, Chmielecki J, Rosenberg M, O'Connor R, Byrne C, Wilder FG, Thorn K, Agius P, Kuk D, Socci ND, Qin L-X, Meyerson M, Hameed $M$, Singer S. Near universal detection of alterations in CTNNB1 and Wnt pathway regulators in desmoid-type fibromatosis by whole-exome sequencing and genomic analysis. Genes Chromosomes Cancer. 2015;54:606-615.

7. Salas S, Chibon F, Noguchi T, Terrier P, Ranchere-Vince D, Lagarde P, Benard J, Forget S, Blanchard C, Dômont J, Bonvalot S, Guillou L, Leroux A, Mechine-Neuville A, Schöffski P, Laë M, Collin F, Verola O, Carbonnelle A, Vescovo L, Bui B, Brouste V, Sobol H, Aurias A, Coindre J-M. Molecular characterization by array comparative genomic hybridization and DNA sequencing of 194 desmoid tumors. Genes Chromosomes Cancer. 2010;NA560-NA568.

8. Larramendy ML, Virolainen M, Tukiainen E, Elomaa I, Knuutila S. Chromosome band $1 \mathrm{q} 21$ is recurrently gained in desmoid tumors. Genes Chromosomes Cancer. 1998;23:183-186.

9. Brandal $P$, Micci $F$, Bjerkehagen $B$, Eknaes $M$, Larramendy $M$, Lothe RA, Knuutila S, Heim S. Molecular cytogenetic characterization of desmoid tumors. Cancer Genet Cytogenet. 2003;146:1-7.
10. Fletcher JA, Naeem R, Xiao S, Corson JM. Chromosome aberrations in desmoid tumors. Trisomy 8 may be a predictor of recurrence. Cancer Genet Cytogenet. 1995;79:139-143.

11. Aretz $\mathrm{S}$, Stienen $\mathrm{D}$, Uhlhaas $\mathrm{S}$, et al. Large submicroscopic genomic APC deletions are a common cause of typical familial adenomatous polyposis. J Med Genet. 2005;42:185-192.

12. Su L-K, Kohlmann W, Ward PA, Lynch PM. Different familial adenomatous polyposis phenotypes resulting from deletions of the entire APC exon 15. Hum Genet. 2002;111:88-95.

13. Dômont J, Salas S, Lacroix L, Brouste V, Saulnier $P$, Terrier $P$, Ranchère $D$, Neuville A, Leroux A, Guillou L, Sciot R, Collin F, Dufresne A, Blay J-Y, Le Cesne A, Coindre J-M, Bonvalot S, Bénard J. High frequency of beta-catenin heterozygous mutations in extra-abdominal fibromatosis: a potential molecular tool for disease management. Br J Cancer. 2010;102:1032-1036.

14. van Broekhoven DLM, Verhoef C, Grünhagen DJ, van Gorp JMHH, den Bakker MA, Hinrichs JWJ, de Voijs CMA, van Dalen T. Prognostic value of CTNNB1 gene mutation in primary sporadic aggressive fibromatosis. Ann Surg Oncol. 2015;22:1464-7015.

15. Jeng YM, Wu MZ, Mao TL, Chang MH, Hsu HC. Somatic mutations of beta-catenin play a crucial role in the tumorigenesis of sporadic hepatoblastoma. Cancer Lett. 2000;152:45-51.

16. Koch A, Denkhaus D, Albrecht S, et al. Childhood hepatoblastomas frequently carry a mutated degradation targeting box of the beta-catenin gene. Cancer Res. 1999;59:269-273.

17. Breuhahn K, Singh S, Schirmacher P, et al. Large-scale N-terminal deletions but not point mutations stabilize beta-catenin in small bowel carcinomas, suggesting divergent molecular pathways of small and large intestinal carcinogenesis. J Pathol. 2008;215:300-307.

\section{SUPPORTING INFORMATION}

Additional supporting information may be found online in the Supporting Information section at the end of the article.

How to cite this article: Colombo C, Urbini M, Astolfi A, et al. Novel intra-genic large deletions of CTNNB1 gene identified in wild type desmoid-type fibromatosis. Genes Chromosomes Cancer. 2018;57:495-503. https://doi.org/10.1002/gcc.22644 